\title{
The Detached Self: Investigating the Effect of Depersonalisation on Self-Bias in the Visual Remapping of Touch
}

\author{
Harry Farmer ${ }^{1,2,3, *}$, Antonio Cataldo ${ }^{4}$, Nagela Adel ${ }^{1}$, Emma Wignall ${ }^{1}$, \\ Vittorio Gallese $^{4,5}$, Ophelia Deroy ${ }^{4,6,7}$, Antonia Hamilton ${ }^{1}$ and \\ Anna Ciaunica ${ }^{1,8}$ \\ ${ }^{1}$ Institute of Cognitive Neuroscience, University College London, Gower Street, London, \\ WC1E 6BT, UK \\ ${ }^{2}$ School of Human Sciences, University of Greenwich, Dreadnought Building, London, SE10 \\ 9LS, UK \\ ${ }^{3}$ Institute for Lifecourse Development, University of Greenwich, Dreadnought Building, \\ Greenwich, London SE10 9LS, UK \\ ${ }^{4}$ Institute of Philosophy, School of Advanced Study, University of London, Senate House, \\ Malet Street, London, WC1E 7HU, UK \\ ${ }^{5}$ Department of Medicine and Surgery, Unit of Neuroscience, University of Parma, Via \\ Volturno 39, 43100 Parma, Italy \\ ${ }^{6}$ Munich Center for Neuroscience, Ludwig Maximilian University, Ludwigstraße 31, 80539 \\ Munich, Germany \\ ${ }^{7}$ Faculty of Philosophy and Philosophy of Science, Ludwig Maximilian University, \\ Geschwister-Scholl-Platz 1, 80539 Munich, Germany \\ ${ }^{8}$ Department of Philosophy, University of Porto, Via Panorâmica s/n, 4150-564 Porto, \\ Portugal
}

Received 3 December 2019; accepted 31 August 2020

\begin{abstract}
There is a growing consensus that our most fundamental sense of self is structured by the ongoing integration of sensory and motor information related to our own body. Depersonalisation (DP) is an intriguing form of altered subjective experience in which people report feelings of unreality and detachment from their sense of self. The current study used the visual remapping of touch (VRT) paradigm to explore self-bias in visual-tactile integration in non-clinical participants reporting high and low levels of depersonalisation experiences. We found that the high-DP group showed
\end{abstract}

\footnotetext{
${ }^{*}$ To whom correspondence should be addressed. E-mail: h.farmer@gre.ac.uk
} 
an increased overall VRT effect but a no-self-face bias, instead showing a greater VRT effect when observing the face of another person. In addition, across all participants, self-bias was negatively predicted by the occurrence of anomalous body experiences. These results indicate disrupted integration of tactile and visual representations of the bodily self in those experiencing high levels of DP and provide greater understanding of how disruptions in multisensory perception of the self may underlie the phenomenology of depersonalisation.

\section{Keywords}

Depersonalisation, self, visual remapping of touch, touch, social cognition

\section{Introduction}

The sense of self lies at the heart of conscious experience, anchoring our disparate perceptions, emotions, thoughts and actions into a unitary whole (Bermúdez, 2002; Metzinger, 2009; Zahavi, 2005). There is a growing consensus that representations of the internal and external states of the body play a key role in constituting and structuring our most basic sense of selfawareness, bodily self-consciousness (Ciaunica and Crucianelli, 2019; Damasio and Damasio, 2006; Farmer and Tsakiris, 2012; Seth and Tsakiris, 2018). Recent work in philosophy, psychology and cognitive neuroscience has linked bodily self-consciousness to the processing of multisensory bodily signals in the brain which need to be integrated in order to obtain an accurate sense of self, world and other (Blanke and Metzinger, 2009; Deroy et al., 2018; Gallagher, 2000; Tsakiris, 2010).

Importantly, multisensory integration is crucial not only for self-representations, but also for self-other distinction and social interactions. For example, viewing touch on another person's body activates brain regions that are typically recruited when perceiving touch on one's own body (Blakemore et al., 2005; Ebisch et al., 2008; Keysers and Gazzola, 2009). These findings suggest the existence of a tactile mirror system analogous to the motor and emotional mirror systems, which have been hypothesised to play a key role in understanding others (Gallese, 2007).

While most of the previous works have studied the link between multisensory integration and bodily self-consciousness under normal conditions (Serino et al., 2008; Tsakiris, 2010), one potentially fruitful route to understanding bodily self-consciousness is to examine conditions in which the ordinary experience of the self becomes disrupted. Here, we investigate two key elements of bodily self-consciousness, namely multisensory integration and self-other differentiation, in a non-clinical population with depersonalisation symptoms. Depersonalisation (DP) is an intriguing form of altered subjective experience in which people report feelings of unreality and detachment from 
their sense of self, as if they were an outside observer of their bodily sensations, thoughts and feelings (Sierra, 2009; Simeon, 2004). While DP experiences are often transitory, in extreme cases they can become chronic leading to a diagnosis of depersonalisation disorder (DPD). Interestingly, those experiencing chronic DP often also report strong feelings of social estrangement (Simeon and Abugel, 2006) and show high levels of social anxiety (Michal et al., 2005). As one sufferer of DPD puts it: 'Nothing makes it [DPD] better but being with other people makes it worse' (Baker et al., 2003, p. 431).

DP has been linked to impaired integration of internal and external representations of the self, which has been argued to play a key role in bodily self-consciousness (Park and Blanke, 2019). Simeon et al. (2000) employed positron emission tomography (PET) to investigate differences between DPD patients and healthy controls and found abnormal activity in parietal and temporal regions involved in multimodal integration, including those involved in the maintenance of body schema. Adler et al. (2016) used EEG to monitor the somatosensory evoked potentials (SEPs) of participants with high and low occurrence of DP experiences while they watched either their own or another person's face being touched. Their high-DP group showed a lack of selfrelated processing advantage in both early and late SEPs. Abnormal self-face processing has also been observed in patients with DPD (Ketay et al. 2014) and with dissociative identity disorder (Lebois et al., 2019).

Further evidence for disrupted bodily self-consciousness in DP comes from two recent studies. O'Sullivan et al. (2018) showed that increased DP experiences were predictive of both the sense of ownership over one's face and the extent to which asynchronous visuomotor stimulation disrupted feelings of agency over facial movements. Dewe et al. (2018) examined the relationship between different dimensions of DP and autonomic response to threats to self or other. They found that scores on the abnormal body experience subscale of the Cambridge Depersonalisation Scale (CDS-29; Sierra and Berrios, 2000) predicted reduced autonomic response for threats to self, while scores on the alienation from surrounding subscale predicted a reduced response for threats to others.

We used the visual remapping of touch paradigm (VRT; Serino et al., 2008) to investigate the dynamics underlying integration of different sources of selfrelevant information in people with DP. In the VRT paradigm a tactile stimulus is delivered to each cheek, one with a higher intensity than the other. This usually leads to the stronger stimulus extinguishing the weaker one from conscious detection. However, if congruent bilateral visual stimuli are observed at the same time this can reduce the extinction effect, leading participants to report detecting both stimuli. A similar effect has also been found for a nonface body part, namely the hands (Gillmeister, 2014), suggesting that the VRT effect generalises to tactile perception in general. Interestingly the VRT effect 
is enhanced by self-relevance with as stronger effect for: one's own compared to another's face (Serino et al., 2008); faces belonging to social in-groups compared to out-groups (Serino et al., 2009) and for humans compared to non-humans (Beck et al., 2013).

This self-enhancement effect appears to depend on a fronto-parietal network integrating information from visual and somatic sensory regions (Cardini et al., 2011). Studies have also shown that VRT effects for other's faces are increased after synchronous visuo-tactile stimulation (Cardini et al., 2013; Fini et al., 2013), a manipulation that has been shown to enhance perceived similarity in both bodily (Tsakiris, 2008) and social (Farmer and Maister, 2017; Farmer et al., 2012; Paladino et al., 2010) domains.

However, it is currently unclear whether dissociation from the self is associated with a decrease or an increase in multisensory integration. On the one hand several studies have indicated evidence in favour of disrupted mirroring of tactile and nociceptive stimuli between self and other in those with high depersonalisation (Adler et al., 2016; Dewe et al., 2018; O'Sullivan et al., 2018; Simeon et al., 2000). However, other recent studies on other types of bodily dissociation, e.g., out of body experiences (OBE; Braithwaite et al., 2017) or the use of dissociative drugs (Morgan et al., 2011) suggest a link between other forms of dissociative experiences and wider temporal windows of multisensory integration, reflected in increased integration when stimuli are presented asynchronously. The current study investigated how the frequency and duration of DP experiences affected the self-face bias in VRT. To do this we first pre-screened participants from the general population in order to divide them into groups with high and low occurrence of DP experiences and then tested these participants using the VRT paradigm.

We hypothesised two key results from this study:

H1. Based on evidence of abnormal integration of bodily signals specifically for people who experienced high levels of DP (Dewe et al., 2018; O'Sullivan et al., 2018; Simeon et al., 2000), we hypothesised that those in the high-DP group would show a reduced VRT effect compared to those in the low-DP group, regardless of the face observed.

H2. Building on evidence from previous studies showing impaired selfprocessing in those with high DP (Adler et al., 2016; Dewe et al., 2018; Ketay et al., 2014; O'Sullivan et al., 2018), we hypothesised that our high-DP group would not show the self-bias effect seen in previous VRT studies. 


\section{Methods}

\subsection{Participants}

In total 63 participants ( 17 male) with a mean age of $24 \pm 4$ participated in this study. Participants were selected for the study after completing an online screening questionnaire which consisted of the CDS-29 and four additional catch questions taken from Adler and colleagues (2016), which were: (1) 'Do you have nightmares more than four times/week?'; (2) 'Are you taking antidepressants or antipsychotics?'; (3) 'Do you see colours when you listen to music?'; (4) 'Are you suffering from a seizure disorder?' These questions served two purposes: firstly they allowed us to remove respondents who defaulted to answering yes to any question they were given. In addition, the use of these specific questions reduced the chance that participants in our high-DP group would also be suffering from comorbid phenomena such as synaesthesia, psychosis or depression. In total 429 people responded to the survey. Respondents who answered yes to any of these four questions were excluded regardless of their score on the CDS-29, leaving a sample of 373 participants. We selected our participants from those who scored a total of 50 or higher (high DP, 25.2\% of respondents) and from those who scored 20 or lower (low DP, $40.75 \%$ of respondents). This cutoff criterion was first used by Kanayama et al. (2009) and meant that our two groups were meaningfully distinct in their levels of DP experiences with the high-group threshold being relatively close to 70 which was shown to yield a sensitivity of $75.5 \%$ for patients with DPD (Sierra et al., 2005). The use of these cutoffs avoided a common limitation of splitting groups based on a pre-determined value or at the median value of the sample, both of which leads to participants at the boundaries of each group having scores that are much closer to each other than they are to the mean of their group.

From the original 63 participants, 30 had high levels of DP experiences (score $\geqslant 50$ on CDS-29) while the other 33 had low levels of DP experiences (score $\leqslant 20$ on the CDS-29). Participants with an overall accuracy of below $20 \%$ or above $90 \%$ in our key trials (in which participant's both observed bilateral finger trajectories and received bilateral tactile stimulation) were excluded due to the likelihood that they were consistently responding with only one of the three options. In addition, we also excluded any participants whose CDS-29 score shifted between the screening and experimental session such that they no longer met the criteria for their original category. Following exclusion our final sample consisted of 40 participants (eight male) with a mean age of $24 \pm 4$. Of these, 18 participants had high and 22 had low levels of DP. The study was approved by the University College London, Institute of Cognitive Neuroscience Research Department's Ethics Committee and complied with the Declaration of Helsinki (2013). A sensitivity analysis run using 
$\mathrm{G}^{*}$ Power 3.1.9.2 (Faul et al., 2007) suggested that, with alpha (i.e., the chance of type- 1 error) set at 0.05 and power (i.e., the chance of type- 2 error) set at 0.8 , this sample size would be sufficient to detect an interaction effect between image type and finger trajectory with a Cohen's $f$ of 0.19 or greater suggesting adequate power to detect a small to medium effect size (Cohen, 1992).

\subsection{Design}

The study employed a mixed design with one between-subjects factor DP group (high vs low) and two within-subjects factors of image type (self, other, ball) and finger trajectory (touch, no touch). The dependent variable was accuracy in detecting bilateral touch.

\subsection{Materials}

\subsubsection{Questionnaires}

Participants' experience of DP was assessed using the CDS-29 (Sierra and Berrios, 2000). The CDS-29 consists of four subscales: anomalous body experiences (ABEs), including feelings of disembodiment, lack of body ownership and lack of agency; emotional numbing (EN); anomalous subjective recall (ASR), particularly when remembering autobiographical events; and alienation from surroundings (AFS), i.e., derealisation (Sierra et al., 2005). Additional measures taken and the analysis of their relationship to VRT accuracy and CDS-29 scores are given in the Supplementary material.

\subsubsection{Experimental Stimuli}

The faces for the other-face used in the study were images of actors with neutral expressions taken from the NimStim face database (Tottenham et al., 2009) which were then modified to add in fingers moving in either a touch or no-touch trajectory (see Fig. 1). Due to previous evidence that similarity to self affects the VRT effect, the other-face was matched for gender and, as far as possible, ethnicity. The NimStim set contains male and female faces from white, black and east Asian ethnic backgrounds but due to the ethinic composition of our sample we also included south Asian faces taken, with permission, from other participants. Participants who did not identify with any of these four ethnicities were shown all four faces during the pre-session and asked to indicate which person they felt looked most similar to themselves.

Transcutaneous electrical nerve stimulation was delivered by means of two Digitimer DS5 constant current stimulators (Digitimer, Ltd., Welwyn Garden City, UK) controlled by a computer. Two pairs of self-adhesive surface electrodes $(20 \times 25 \mathrm{~mm}$ Disposable solid gel, Unimed, Farnham, UK) connected to the stimulator were placed on the left and right check of participants. Electrical impedance between each electrode and the skin was kept below $5 \mathrm{k} \Omega$ 
throughout the experiment. Visual stimuli for instructions and tasks were delivered using Psychtoolbox 3 (Brainard, 1997; Kleiner et al., 2007; Pelli, 1997) run on Matlab 17b (Mathworks, 2017).

\subsection{Procedure}

\subsubsection{Pre-Experiment Session}

Participants attended a pre-experiment session in which they were photographed with a neutral expression against a coloured background whilst wearing a uniform grey t-shirt and with long hair tied back so as not to conceal the face. They were also asked to complete the four additional questionnaires (see Supplementary material; see Fig. 1A). The image of the participant's face was then edited by resizing and extracting the face from the original photo and placing it on a white background using GIMP (The GIMP team, 2017). The faces were then rescaled, and the hand images added using a custom Matlab script.

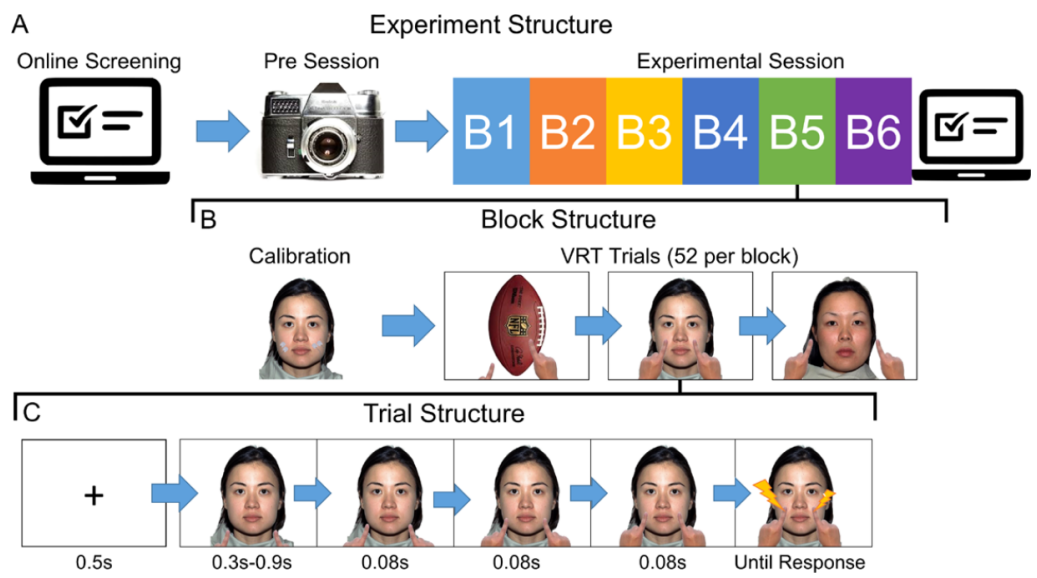

Figure 1. Outline of experimental structure. (A) Participants were selected for high or low depersonalisation (DP) via online screening using the CDS-29. They then attended a preexperimental session in which an image of their face was taken, and they completed a series of individual-differences questionnaires. Finally, they returned for the experimental session during which they completed six experimental blocks before rating the face of the other person and retaking the CDS-29. (B) In each block of the main task participants first completed a calibration task to find their tactile thresholds. They then saw 52 trials of the visual remapping of touch (VRT) task. Here we show examples of a unilateral touch ball trial, a bilateral touch self trial and a bilateral no touch other trial. (C) In each trial participants first saw a fixation cross, they then saw the face/ball and after a variable amount of time a view made of four frames of 0.08 seconds in which one or both hands would either move to touch the face or to touch empty space beside the face. On the final frame tactile stimulation was delivered at an intensity threshold on one cheek (in this case right) and $1.4 \times$ threshold on the other (in this case left). 


\subsubsection{Experimental Session}

Visual Remapping of Touch Task. The VRT task consisted of six blocks. Each block consisted of 52 trials. Participants saw a combination of videos in which they saw either: (a) their own face; (b) the face of a gender- and ethnicitymatched other person; or (c) an American football (see Fig. 1B). Stimulation intensity was calibrated at the start of each block using a staircase procedure (see Supplementary material). The picture of the face $\backslash$ ball was presented as a central, static image in the background of the movie. In the foreground, however, two fingers were presented, initially positioned on the lower part of the screen, one on the right side and one on the left side.

In each trial participants first saw a white fixation cross for $500 \mathrm{~ms}$ and then the image of the face/ball appeared. After a variable interval of $300 \mathrm{~ms}, 600 \mathrm{~ms}$ or $900 \mathrm{~ms}$ the fingers moved toward the face. In different trials, the finger motion followed one of two trajectories. In the touch condition, the fingers touched the cheeks of the shown face, in approximately the same place where tactile stimulation was administered on the participants' cheeks. In the notouch condition, the fingers moved away from the face and stopped about $5 \mathrm{~cm}$ alongside the face (see Fig. 1). In different trials, either the finger on the right, the left, or both fingers moved. This trajectory took $240 \mathrm{~ms}$ to complete and at completion participants received tactile stimulation on one or both cheeks. They were then asked to determine whether they had been touched on the left, right or both cheeks by pressing either the left, right or up arrow key with their right hand. There was no time limit for participants to respond but they were asked to respond as quickly and accurately as possible (see Fig. 1C).

Participants experienced all combinations of unilateral and bilateral finger trajectories and unilateral and bilateral touch. Since the VRT effect is quantified by comparing trials in which bilateral touch is both observed and received, we included a larger number of these trials than the other possible combination. In total, for each type of face stimulus participants saw 30 bilateral touch with bilateral stimulation trials, 24 bilateral no touch with bilateral stimulation trials and three of every other possible combination of trials with a total of 306 trials. Because tactile thresholds are known to show rapid adaptation (Graczyk et al., 2018), we chose to ascertain participants' tactile threshold every 50 trials. Therefore, the full set of conditions were presented across every pair of blocks in a randomised order.

Post VRT Questionnaires. After completing the VRT task participants were asked to complete a series of ratings about the other person (see Supplementary material for details). Finally, participants completed a follow-up version of the CDS-29. Once this survey was completed participants were debriefed, paid for their participation and left the lab. 


\section{Results}

\subsection{Amount of Depersonalisation Experience Interacts With Face Identity to} Modulate Visual Remapping of Touch

In order to investigate whether the high- and low-DP groups differed in the amount of VRT in response to self and other faces, a three-way mixed analysis of variance (ANOVA) was used to test for interaction effects between image type (self, other, ball), finger trajectory (touch, no touch) and DP group (high, low) on accuracy in perceiving bilateral tactile stimulation when viewing bilateral finger trajectories (see Table 1 and Fig. 2). The ANOVA revealed a significant effect of finger trajectory, $F_{(1,38)}=14.99, p<0.001 \eta_{p}^{2}=0.283$ because participants were more accurate in touch compared to no-touch trials. We also found a main effect of DP group, $F_{(1,38)}=5.93, p=0.02$, $\eta_{p}^{2}=0.135$, because the high-DP group were more accurate than the lowDP group. The main effect of image type was not significant, $F_{(2,76)}=1.63$, $p=0.202, \eta_{p}^{2}=0.041$.

In addition to these main effects we also found a significant two-way interaction between finger trajectory and DP group, $F_{(1,38)}=4.99, p=0.032$, $\eta_{p}^{2}=0.116$. Post-hoc testing using pairwise comparisons of the estimated marginal means with Bonferroni-adjusted levels revealed that the high-DP group were significantly more accurate in touch compared to no-touch trials, $p^{\prime}$ (adjusted $p$ value $)<0.001$. In contrast, there was no significant difference

\section{Table 1.}

Means and standard deviations of accuracy in responding to bilateral tactile stimulation when viewing bilateral finger trajectories by depersonalisation (DP) group, finger trajectory and image type

\begin{tabular}{lcccc}
\hline Group & Trajectory & Image & Mean & SD \\
\hline High DP & Touch & Self & $58.7 \%$ & $18.93 \%$ \\
& & Other & $65 \%$ & $21.94 \%$ \\
& Ball & $62.22 \%$ & $21.27 \%$ \\
& No Touch & Self & $55.56 \%$ & $23.13 \%$ \\
& & Other & $54.17 \%$, & $19.44 \%$ \\
& Ball & $55.32 \%$ & $18.73 \%$ \\
Low DP & Touch & Self & $44.09 \%$ & $18.13 \%$ \\
& & Other & $43.33 \%$ & $17.73 \%$ \\
& No Touch & Ball & $48.48 \%$ & $18.89 \%$ \\
& & Self & $41.66 \%$ & $19.88 \%$ \\
& & Other & $45.83 \%$ & $20.89 \%$ \\
& & Ball & $42.8 \%$ & $19.21 \%$ \\
\hline
\end{tabular}




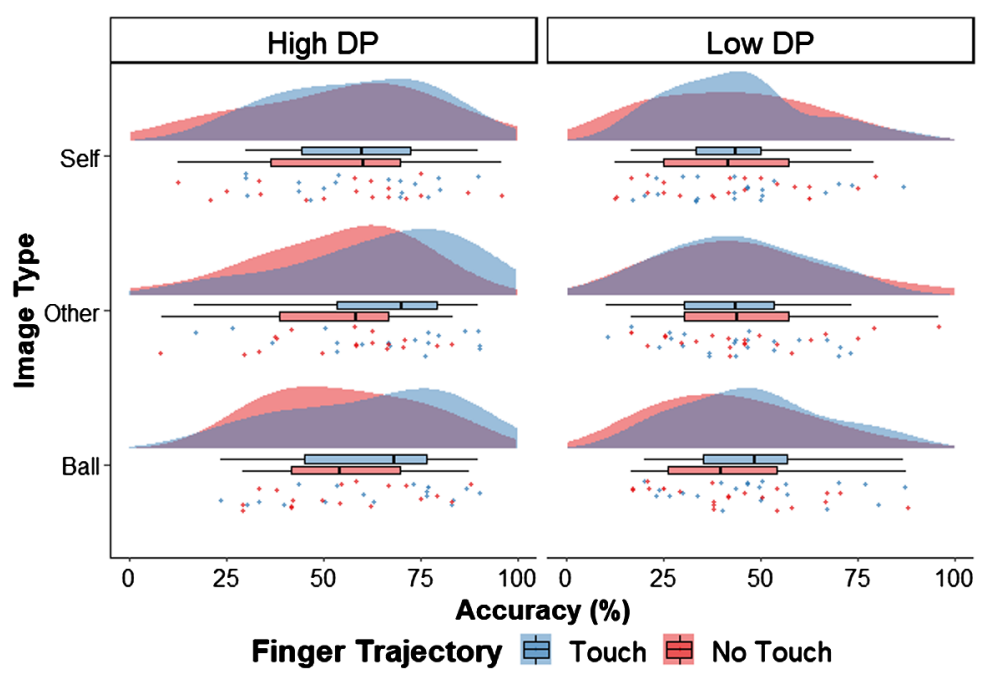

Figure 2. Raincloud plot of accuracy in detecting bilateral touch across finger trajectory, image type and depersonalisation (DP) group. Clouds represent distribution, raindrops represent individual participants, bars represent $95 \%$ confidence intervals.

between touch and no-touch trials for the low-DP group, $p^{\prime}=0.229$. No other two-way interactions were found to be significant.

However, this effect was qualified by a significant three-way interaction between image type, finger trajectory and DP group, $F_{(2,76)}=3.76, p=$ $0.028, \eta_{p}^{2}=0.09$. Bonferroni-adjusted pairwise comparisons of the estimated marginal means revealed that this effect was driven by the high-DP group having significantly greater accuracy in touch compared to no-touch trials for the other-face, $p^{\prime}=0.001$, and for the ball, $p^{\prime}=0.015$, but not for the self-face, $p^{\prime}=0.259$ In contrast the low-DP group did not show a significant difference in accuracy between touch and no touch for either the self-face, $p^{\prime}=0.336$, or the other-face, $p^{\prime}=0.352$ but did show a difference for the ball, $p^{\prime}=0.026$.

\subsection{Individual Variance in Amount of Anomalous Body Experiences Predicts Self-Bias in VRT}

To further investigate this effect with a focus on differences in self- and otherface processing between participants with high- and low-DP experiences, we calculated VRT indexes for both self and other faces by subtracting accuracy in the no-touch condition from that in the touch condition. We then created a VRT self-bias index by subtracting the other VRT index from the self-VRT index. We then carried out a four-stage hierarchical multiple regression with VRT self-bias as the dependent variable and scores on the four CDS-29 subscales from the experimental session as predictors. The subscales were entered in an order that seemed plausible given their relationship to self-perception. As 
our task related to processing bodily stimuli, the anomalous body experience subscale was entered at stage one of the regression. Anomalous subjective recall was entered at stage two, due to its relation to memories of the self and self-face. Emotional numbing was entered at stage three due to the relationship between emotional experiences and somatic processing (Damasio and Carvalho, 2013). Finally, we added AFS at stage four as this was the subscale that was least related to self and body processing.

Full results of the hierarchical regression are given in Table 2. As can be seen, the amount of ABEs reported was a significant negative predictor of selfbias in VRT, $R^{2}=0.134, F_{(1,38)}=5.9, p=0.02$ (see Fig. 3). The additional other three subscales did not lead to significant improvements in model fit.

In addition to this analysis we also investigated the role of individual variation in DP traits in predicting overall VRT by running the same model but with the average of VRT scores across all images as the dependent variable. Full results of that hierarchical regression are given in Supplementary Table S1. However, the key finding was that the model was significant at the second level, $R^{2}=0.15, F_{(1,38)}=4.64, p=0.049$. In this model anomalous subject recall was a significant positive predictor of overall VRT, $\beta=0.629$, $p=0.038$, while anomalous body experience was a non-significant negative predictor, $\beta=-0.328, p=0.269$. The addition of the other two subscales did not lead to significant improvements in model fit.

Table 2.

Summary of Hierarchical Regression Analysis for CDS-29 subscales predicting VRT self-bias

\begin{tabular}{lrccccr}
\hline Variable & $\beta$ & $p$ & $R^{2}$ & $\Delta R^{2}$ & $\Delta \mathrm{F}$ & $\Delta \mathrm{p}$ \\
\hline Step 1 & & & 0.134 & 0.134 & 5.9 & 0.02 \\
Anomalous Body Experience & -0.367 & 0.02 & & & & \\
Step 2 & & & 0.145 & 0.11 & 0.46 & 0.5 \\
Anomalous Body Experience & -0.196 & 0.507 & & & & \\
Anomalous Subjective Recall & -0.199 & 0.500 & & & & \\
Step 3 & & & 0.194 & 0.49 & 2.18 & 0.149 \\
Anomalous Body Experience & -0.445 & 0.191 & & & & \\
Anomalous Subjective Recall & -0.266 & 0.368 & & & & \\
Emotional Numbing & 0.379 & 0.149 & & & & \\
Step 4 & & & 0.215 & 0.21 & 0.92 & 0.344 \\
Anomalous Body Experience & -0.586 & 0.117 & & & & \\
Anomalous Subjective Recall & -0.336 & 0.271 & & & & \\
Emotional Numbing & 0.232 & 0.444 & & & & \\
Alienation from Surroundings & 0.364 & 0.344 & & & &
\end{tabular}




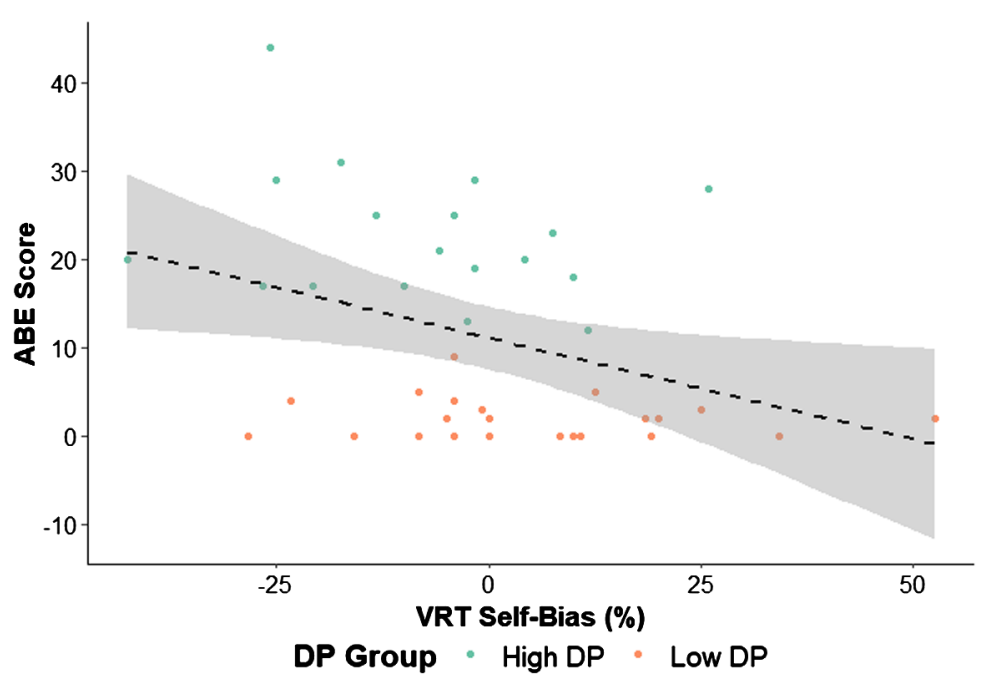

Figure 3. Negative linear relationship between self-bias in visual remapping of touch (VRT) and anomalous body experience (ABE) scores. DP, depersonalisation.

\subsection{Low-Depersonalisation Group Showed Reduced Sensitivity for Self Faces but Higher Criterion for Bilateral Touch}

Although the small number of unilateral trials in the study means that our experimental design is not optimised for signal detection theory analysis, we followed Cardini et al. (2013) in investigating whether the observed differences between groups when perceiving bilateral touch were related to changes in sensitivity or in response criterion. We, therefore, calculated sensitivity $\left(d^{\prime}\right)$ and criterion $(C)$ scores across all trials where participants saw bilateral finger movements. To do this we classified: (1) a 'bilateral' response to bilateral stimulation as a hit; (2) a unilateral (left or right) response to a bilateral stimulation as an omission; (3) a left response to a left stimulation or a right response to a right stimulation as a correct rejection; and (4) a bilateral response to unilateral (left or right) stimulation as a false alarm. Separate $d^{\prime}$ and $C$ scores were generated for the touch and no-touch conditions of each image type.

Two separate mixed ANOVA, one for $d^{\prime}$ and one for $C$ scores, were carried out with image type (self, other, ball), finger trajectory (touch, no touch) and amount of DP experiences (high-DP group, low-DP group) as the factors. For $d^{\prime}$, there were no significant main effects, but there was a significant interaction between image type and amount of DP experiences, $F_{(2,76)}=5.28, p=$ 0.007 . Bonferroni-adjusted pairwise comparisons of the estimated marginal means revealed that this effect was driven by the low-DP group having significantly lower sensitivity than the high-DP group for the self-face condition, 
Table 3.

Means and standard deviations of $d^{\prime}$ in responding to bilateral tactile stimulation when viewing bilateral finger trajectories by depersonalisation (DP) group and image type

\begin{tabular}{lccc}
\hline Group & Face & Mean & SD \\
\hline High DP & Self & 1.27 & 0.72 \\
& Other & 1.36 & 0.94 \\
& Ball & 1.27 & 0.81 \\
Low DP & Self & 0.79 & 0.72 \\
& Other & 0.99 & 0.69 \\
& Ball & 1.08 & 0.66 \\
\hline
\end{tabular}

$p^{\prime}=0.022$. However, the two groups did not significantly differ in sensitivity for the other-face, $p^{\prime}=0.193$, or the ball conditions, $p^{\prime}=0.54$. No other interactions were significant (see Table 3).

The ANOVA on $C$ scores revealed a significant main effect of image type, $F_{(2,76)}=4.85, p=0.01$. Bonferroni-adjusted pairwise comparisons of the estimated marginal means revealed that this effect was driven by a significantly higher criterion for the ball image $(\mathrm{EMM}=0.58, \mathrm{SE}=0.05)$ compared to either the self-face image $\left(\mathrm{EMM}=0.48, \mathrm{SE}=0.04, p^{\prime}=0.009\right)$ or the otherface image $\left(\mathrm{EMM}=0.5, \mathrm{SE}=0.05, p^{\prime}=0.01\right)$. In addition, we found a main effect of group, $F_{(1,38)}=6.01, p=0.019$. This was because the criterion for the high-DP group $(\mathrm{EMM}=0.42, \mathrm{SE}=0.32)$ was significantly smaller than that of the low-DP group $(\mathrm{EMM}=0.62, \mathrm{SE}=0.21)$. No other significant main effects or interactions were found. A follow-up analysis demonstrated that these effects were not due to significant differences in tactile thresholds between the two groups (see Supplementary material for details).

\section{Discussion}

The current study investigated whether the experience of depersonalisation (DP), a condition in which people report subjective feelings of self-detachment and estrangement, modulated the VRT effect. Due to the alterations in selfexperience that characterise DP, we predicted that participants in our high-DP group would show a reduced overall VRT effect regardless of the observed face (H1) and fail to show the self-bias VRT effect previously reported in other studies (H2). Our results offered support for $\mathrm{H} 2$ by showing that amount of ABEs reported by participants was negatively correlated with the size of their VRT self-bias. However, contrary to H1, we found that the high-DP group showed a VRT effect in the other-face condition while the low-DP group did not show a VRT effect in either the self- or - conditions. 


\subsection{Effects of Depersonalisation on Visual Remapping of Touch}

Despite previous evidence for disruption in attentional and perceptual processing in people experiencing DP (Giesbrecht et al., 2008; Guralnik et al., 2000), our high-DP group had greater overall accuracy in the detection of bilateral touch. In addition, they showed a VRT effect for faces that was lacking in our low-DP group, suggesting an increased tendency to integrate visual and tactile information together during the task, which goes against previous evidence for disrupted activity in multisensory integration regions in DPD (Simeon et al., 2000).

One possible explanation for this discrepancy comes from previous studies that suggest a significant link between experiences of depersonalisation and dissociation and increased tendency to integrate multisensory information. Kanayama et al. (2009) used the same screening criteria as the current study to identify low- and high-DP groups and showed that the high-DP group was considerably more susceptible to the rubber hand illusion (RHI) than the low-DP group. Similarly, Braithwaite et al. (2017) demonstrated that participants disposed to OBEs, who also scored high in the ABE and AFS subscales of the CDS-29, reported increased body ownership after asynchronous visualtactile integration in the RHI. Similar overintegration of visuotactile stimuli has been found in participants who had taken the dissociative drug ketamine (Morgan et al., 2011). Finally, a recent study from Bowling et al. (2019) found a strong positive relationship between trait depersonalisation and the tendency to experience pain on their own body when viewing others in pain (mirror-pain synaesthesia).

These findings suggest that, contrary to our initial hypothesis, those high in depersonalisation tend to over-, rather than underintegrate multisensory signals related to self and non-self objects. This may be due to the fact that they have decreased sensory precision when detecting their own bodily states leading to a greater reliance on external cues (Seth et al., 2011; Tajadura-Jiménez and Tsakiris, 2014) Applied to the current study this account explains both the finding that participants in our high-DP group showed a stronger VRT effect and the fact that overall mean VRT was positively predicted by the ASR subscale of the CDS. Qualified support for the idea that participants high in DP may have relied more on external cues as opposed to showing a greater ability to accurately detect bilateral stimuli comes from our sensitivity analysis. This suggests that the higher overall accuracy in the high-DP group was not due to increased sensitivity but rather that they had a significantly lower criterion for reporting bilateral touch. Since, in line with previous VRT studies (Beck et al., 2013; Cardini et al., 2013; Serino et al., 2009), we only examined trails in which participants received bilateral tactile stimulation, this increased tendency to report bilateral stimulation regardless of the actual stimulation would 
automatically result in increased accuracy. However, it should be noted that this difference in criterion did not interact with finger trajectory, meaning that it cannot fully explain the difference between the two groups in VRT.

The link between high levels of depersonalisation and increased integration of multisensory signals may also explain why our low-DP group did not show a strong VRT effect in any of our stimuli conditions. We followed Adler et al. (2016) in defining our low-DP group as participants with a CDS score of below 20. However, this group only made up $40 \%$ of the total number of people surveyed during screening, making it probable that they also made up only around the same proportion of participants in previous VRT studies. If higher rates of DP are related to a greater general tendency to integrate multisensory information, then it is plausible that our low-DP group was made up of those least susceptible to the interference of external signals when making tactile judgements. In other words, by selecting for low-DP experience we may have inadvertently recruited a group that was relatively unsusceptible to the VRT effect in comparison to more heterogenous samples recruited in previous VRT studies.

Surprisingly, despite the lack of a VRT effect in the low-DP group overall and the lack of a VRT effect for self faces in the high-DP group we also found significant VRT for the ball condition in both the high- and low-DP groups. This result was unexpected since previous VRT studies have failed to find evidence of remapping of touch onto non-face objects including both balls and houses. However, there is some evidence from previous neuroimaging studies that neural regions involved in perceiving touch including the secondary somatosensory cortex are also activated when people observe non-human objects (e.g., rolls of paper) being touched compared to when they are not touched (Keysers et al., 2004). Why both our groups showed the VRT effect for this non-face condition but not for the two face conditions is unclear and this unexpected result should be further explored further in future research. For the rest of this paper we will focus on the contrasting findings between the groups for the VRT on self and other faces.

\subsection{Effect of Depersonalisation on Self-Bias in Visual Remapping of Touch}

While we found that the high-DP group did show significantly greater VRT effects than the low-DP group it is worth noting that this effect was qualified by a three-way interaction between DP group, finger trajectory and face type. This was driven by the fact that the high-DP group showed a significant VRT effect in the other-face and ball conditions but not in the self-face condition. This finding supports our second hypothesis that people with a higher rate of DP experiences do not show the typical pattern of self-face bias when integrating visual-tactile information. Instead our high-DP group showed the reverse 
pattern with greater remapping for the other than for the self. We further investigated this effect by running a hierarchical regression model on the difference in VRT effect between self- and other-face conditions with scores on the CDS subscales as predictors. This regression indicated a negative relationship between VRT self-bias scores and the ABE subscale suggesting that the lack of self-bias seen in our high-DP group was specifically related to the bodily component of DP.

It is also worth noting that our sensitivity analysis revealed a significant effect of DP group by image type. Post-hoc tests indicated that this was due to the low-DP group having lower sensitivity than the high-DP group when detecting bilateral touch for the self-face image. In contrast there was no difference in sensitivity between the two groups for the other-face or ball images. To our knowledge this is the first VRT study to find such a sensitivity effect which may help to explain the mechanism by which the VRT self-bias effect occurs, i.e., via a reduction in sensitivity to tactile information and increased reliance on visual information when observing one's own face compared to the faces of others. However it should be noted that a previous sensitivity analysis using VRT data, albeit with a smaller number of trials, did not find any evidence for a self-bias in sensitivity (Cardini et al., 2013) and further research with a design better suited to full sensitivity analyses would be needed to confirm this.

This result adds to previous findings indicating a disruption to self-related processing in people with high DP experiences, especially in terms of the integration of internal and external information about the bodily self (Adler $e t$ al., 2016; Dewe et al., 2018; Ketay et al., 2014; O'Sullivan et al., 2018). It complements Adler and colleagues' finding of anomalous neural integration of self-related visuotactile stimuli by providing evidence that high DP is reflected behaviourally in (a) the abnormal remapping of self-related information and (b) a lack of any self-related enhancement in sensitivity in tactile detection. We further add to this literature by showing that this behavioural effect is specifically related to the extent to which participants experienced abnormal sensations relating to their own body.

One interesting question is whether the lack of the typical self-bias in VRT found in those with a high occurrence of DP is due to bottom-up impairments in multisensory integration of self-related information or whether this process is driven by top-down inhibition. In the first case the lack of a VRT effect for the self would be closely related to the findings of anomalous processing in brain regions involved in sensory integration (Simeon et al., 2000) or in attentional processing (Adler et al., 2014; Giesbrecht et al., 2008; Guralnik et al., 2000; Schabinger et al., 2018) in DP. On the second account, advantages due to self-processing may be inhibited due to conflict between explicit selfrecognition and feelings of detachment from the observed self-face. On this 
account disrupted processing when observing the self-face would occur in an analogous way to the manner in which frontal regions like the DLPFC have been related to the emotional numbing that comprises another key aspect of depersonalisation (De Andrade et al., 2016; Sierra and David, 2011). While further research is necessary to provide a definitive answer to this question, for now we note that the fact our high-DP group showed a significant VRT effect for the other-face suggests that the lack of an effect for the self-face is less likely to be related to a generalised impairment in multisensory integration or attentional processing and more like to be due to top-down disruption of self-processing.

\subsection{Limitations and Future Directions}

One caveat to the current study is the fact that overall accuracy scores were lower than is commonly reported in VRT studies, over all conditions and both groups the average accuracy was only around $50 \%$ compared to previous reports of accuracy of 58\% (Cardini et al., 2012) or 66\% (Serino et al., 2008), although other studies have reported similar levels of overall accuracy (Serino et al., 2009). It is likely that the lower accuracy seen in the current study reflects the fact that we used a staircase procedure to find the point of subjective equality giving us a tactile threshold of $50 \%$ as opposed to previous studies which set their thresholds at 60\% (Cardini et al., 2012; Serino et al., 2009) or $70 \%$ (Serino et al., 2008). We chose to use this approach as it enabled a more robust and principled means of deriving stimulation at each participant's threshold level of detection. However, this does seem to have led to an unwanted floor effect with significantly lower overall accuracy than most other VRT studies.

Further research in this area could seek to further examine the relationship between DP and mirroring of others' emotional expressions, for example by examining the mechanisms underlying implicit social (self-other) mechanisms such as automatic facial mimicry (Bourgeois and Hess, 2008; Farmer et al., 2018). In addition, a more refined conceptual toolbox describing the phenomenology of DP experiences from a first-person perspective needs to be developed in order to fully capture the complexity and richness of this phenomenon.

\section{Conclusion}

The current study examined the relationship between depersonalisation and self-bias in the integration of visual and tactile stimuli using the VRT task in a non-clinical sample. Our findings revealed that participants high in DP showed an increased overall VRT effect but a no-self-face bias, instead showing a greater VRT effect when observing the face of another person. In addition, 
across all participants, self-bias was negatively predicted by score on the ABEs subscale suggesting that this effect was specifically linked to disruptions in the perception of the bodily self. These results provide evidence for disrupted integration of tactile and visual representations of the bodily self in those experiencing DP and provide a greater understanding of how disruptions in sensory perception of the self may underlie the phenomenology of depersonalisation.

\section{Acknowledgements}

This work was supported by the Fundação Bial (http://bial.com/) grant 157/16.

Dr Cataldo's contribution was supported by an award of the 2017 Summer Seminars in Neuroscience and Philosophy (SSNAP — Duke University), funded by the John Templeton Foundation.

\section{Supplementary Material}

Supplementary material is available online at: https://doi.org/10.6084/m9.figshare.13032395

\section{References}

Adler, J., Beutel, M. E., Knebel, A., Berti, S., Unterrainer, J. and Michal, M. (2014). Altered orientation of spatial attention in depersonalization disorder, Psychiatry Res. 216, 230-235. DOI:10.1016/j.psychres.2014.02.021.

Adler, J., Schabinger, N., Michal, M., Beutel, M. E. and Gillmeister, H. (2016). Is that me in the mirror? Depersonalisation modulates tactile mirroring mechanisms, Neuropsychologia $\mathbf{8 5}$, 148-158. DOI:10.1016/j.neuropsychologia.2016.03.009.

Baker, D., Hunter, E., Lawrence, E., Medford, N., Patel, M., Senior, C., Sierra, M., Lambert, M. V., Phillips, M. L. and David, A. S. (2003). Depersonalisation disorder: clinical features of 204 cases, Br. J. Psychiatry 182, 428-433. DOI:10.1192/bjp.182.5.428.

Beck, B., Bertini, C., Scarpazza, C. and Làdavas, E. (2013). Observed touch on a non-human face is not remapped onto the human observer's own face, PLoS One 8, e73681. DOI:10. 1371/journal.pone.0073681.

Bermúdez, J. L. (2002). The sources of self-consciousness, Proc. Aristotelian Soc. 102, 87-107. DOI:10.1111/j.0066-7372.2003.00044.x.

Blakemore, S.-J., Bristow, D., Bird, G., Frith, C. and Ward, J. (2005). Somatosensory activations during the observation of touch and a case of vision-touch synaesthesia, Brain 128, 15711583. DOI: 10.1093/brain/awh500.

Blanke, O. and Metzinger, T. (2009). Full-body illusions and minimal phenomenal selfhood, Tr. Cogn. Sci. 13, 7-13. DOI:10.1016/j.tics.2008.10.003.

Bourgeois, P. and Hess, U. (2008). The impact of social context on mimicry, Biol. Psychol. 77, 343-352. DOI:10.1016/j.biopsycho.2007.11.008.

Bowling, N. C., Botan, V., Santiesteban, I., Ward, J. and Banissy, M. J. (2019). Atypical bodily self-awareness in vicarious pain responders, Philos. Trans. R. Soc. B: Biol. Sci. 374, 20180361. DOI:10.1098/rstb.2018.0361. 
Brainard, D. H. (1997). The psychophysics toolbox, Spat. Vis. 10, 433-436. DOI:10.1163/ 156856897 X00357.

Braithwaite, J. J., Watson, D. G. and Dewe, H. (2017). Predisposition to out-of-body experience $(\mathrm{OBE})$ is associated with aberrations in multisensory integration: psychophysiological support from a "rubber-hand illusion" study, J. Exp. Psychol. Hum. Percept. Perform. 43, 1125-1143. DOI:10.1037/xhp0000406.

Cardini, F., Costantini, M., Galati, G., Romani, G. L., Làdavas, E. and Serino, A. (2011). Viewing one's own face being touched modulates tactile perception: an fMRI study, J. Cogn. Neurosci. 23, 503-513. DOI:10.1162/jocn.2010.21484.

Cardini, F., Bertini, C., Serino, A. and Ladavas, E. (2012). Emotional modulation of visual remapping of touch, Emotion 12, 980-987. DOI:10.1037/a0027351.

Cardini, F., Tajadura-Jiménez, A., Serino, A. and Tsakiris, M. (2013). It feels like it's me: interpersonal multisensory stimulation enhances visual remapping of touch from other to self, J. Exp. Psychol. Hum. Percept. Perform. 39, 630-637. DOI:10.1037/a0031049.

Ciaunica, A. and Crucianelli, L. (2019). Minimal self-awareness: from within a developmental perspective, J. Consc. Stud. 26, 207-226.

Cohen, J. (1992). A power primer, Psychol. Bull. 112, 155-159. DOI:10.1037/0033-2909.112. 1.155.

Damasio, A. and Carvalho, G. B. (2013). The nature of feelings: evolutionary and neurobiological origins, Nat. Rev. Neurosci. 14, 143-152. DOI:10.1038/nrn3403.

Damasio, A. and Damasio, H. (2006). Minding the body, Daedalus 135, 15-22.

De Andrade, A. P. M., Amaro, E., Farhat, S. C. L. and Schvartsman, C. (2016). Higher burnout scores in paediatric residents are associated with increased brain activity during attentional functional magnetic resonance imaging task, Acta Paediatr. 105, 705-713. DOI:10.1111/ apa.13371.

Deroy, O., Chen, Y.-C. and Spence, C. (2018). Multisensory constraints on awareness, Philos. Trans. R. Soc. B: Biol. Sci. 369, 20130207. DOI:10.1098/rstb.2013.0207.

Dewe, H., Watson, D. G., Kessler, K. and Braithwaite, J. J. (2018). The depersonalized brain: new evidence supporting a distinction between depersonalization and derealization from discrete patterns of autonomic suppression observed in a non-clinical sample, Consc. Cogn. 63, 29-46. DOI:10.1016/j.concog.2018.06.008.

Ebisch, S. J. H., Perrucci, M. G., Ferretti, A., Del Gratta, C., Romani, G. L. and Gallese, V. (2008). The sense of touch: embodied simulation in a visuotactile mirroring mechanism for observed animate or inanimate touch, J. Cogn. Neurosci. 20, 1611-1623. DOI:10.1162/jocn. 2008.20111.

Farmer, H. and Maister, L. (2017). Putting ourselves in another's skin: using the plasticity of self-perception to enhance empathy and decrease prejudice, Soc. Justice Res. 30, 323-354. DOI:10.1007/s11211-017-0294-1.

Farmer, H. and Tsakiris, M. (2012). The bodily social self: a link between phenomenal and narrative selfhood, Rev. Philos. Psychol. 3, 125-144. DOI:10.1007/s13164-012-0092-5.

Farmer, H., Tajadura-Jiménez, A. and Tsakiris, M. (2012). Beyond the colour of my skin: how skin colour affects the sense of body-ownership, Consc. Cogn. 21, 1242-1256. DOI:10. 1016/j.concog.2012.04.011.

Farmer, H., Ciaunica, A. and Hamilton, A. F. de C. (2018). The functions of imitative behaviour in humans, Mind Lang. 33, 378-396. DOI:10.1111/mila.12189. 
Faul, F., Erdfelder, E., Lang, A.-G. and Buchner, A. (2007). G*Power 3: a flexible statistical power analysis program for the social, behavioral, and biomedical sciences, Behav. Res. Meth. 39, 175-191. DOI:10.3758/BF03193146.

Fini, C., Cardini, F., Tajadura-Jiménez, A., Serino, A. and Tsakiris, M. (2013). Embodying an outgroup: the role of racial bias and the effect of multisensory processing in somatosensory remapping, Front. Behav. Neurosci. 7, 165. DOI:10.3389/fnbeh.2013.00165.

Gallagher, S. (2000). Philosophical conceptions of the self: implications for cognitive science, Tr. Cogn. Sci. 4, 14-21. DOI:10.1016/S1364-6613(99)01417-5.

Gallese, V. (2007). Before and below "theory of mind": embodied simulation and the neural correlates of social cognition, Philos. Trans. R. Soc. B: Biol. Sci. 362, 659-669. DOI:10. 1098/rstb.2006.2002.

Giesbrecht, T., Lynn, S. J., Lilienfeld, S. O. and Merckelbach, H. (2008). Cognitive processes in dissociation: an analysis of core theoretical assumptions, Psychol. Bull. 134, 617-647. DOI:10.1037/0033-2909.134.5.617.

Gillmeister, H. (2014). A new perceptual paradigm to investigate the visual remapping of others' tactile sensations onto one's own body shows "mirror touch" for the hands, Front. Psychol. 5, 95. DOI:10.3389/fpsyg.2014.00095.

Graczyk, E. L., Delhaye, B. P., Schiefer, M. A., Bensmaia, S. J. and Tyler, D. J. (2018). Sensory adaptation to electrical stimulation of the somatosensory nerves, J. Neural Eng. 15, 0469002. DOI: 10.1088/1741-2552/aab790.

Guralnik, O., Schmeidler, J. and Simeon, D. (2000). Feeling unreal: cognitive processes in depersonalization, Am. J. Psychiatry 157, 103-109. DOI:10.1176/ajp.157.1.103.

Kanayama, N., Sato, A. and Ohira, H. (2009). The role of gamma band oscillations and synchrony on rubber hand illusion and crossmodal integration, Brain Cogn. 69, 19-29. DOI:10. 1016/j.bandc.2008.05.001.

Ketay, S., Hamilton, H. K., Haas, B. W. and Simeon, D. (2014). Face processing in depersonalization: an fMRI study of the unfamiliar self, Psychiatry Res. Neuroimaging 222, 107-110. DOI:10.1016/j.pscychresns.2014.02.003.

Keysers, C. and Gazzola, V. (2009). Expanding the mirror: vicarious activity for actions, emotions, and sensations, Curr. Opin. Neurobiol. 19, 666-671. DOI:10.1016/j.conb.2009.10. 006.

Keysers, C., Wicker, B., Gazzola, V., Anton, J.-L., Fogassi, L. and Gallese, V. (2004). A touching sight: SII/PV activation during the observation and experience of touch, Neuron 42, 335-346. DOI:10.1016/s0896-6273(04)00156-4.

Kleiner, M., Brainard, D. H. and Pelli, D. (2007). What's new in Psychtoolbox-3, Perception 36 ECVP Abstract Suppl., 1-16.

Lebois, L. A. M., Wolff, J. D., Hill, S. B., Bigony, C. E., Winternitz, S., Ressler, K. J. and Kaufman, M. L. (2019). Preliminary evidence of a missing self bias in face perception for individuals with dissociative identity disorder, J. Trauma Dissociation 20, 140-164. DOI:10. 1080/15299732.2018.1547807.

Mathworks (2017). Matlab R2017b. Retrieved from https://uk.mathworks.com/products/ matlab.html.

Metzinger, T. K. (2009). The Ego Tunnel: the Science of the Mind and the Myth of the Self. Basic Books, New York, NY, USA. 
Michal, M., Kaufhold, J., Grabhorn, R., Krakow, K., Overbeck, G. and Heidenreich, T. (2005). Depersonalization and social anxiety, J. Nerv. Ment. Dis. 193, 629-632. DOI:10.1097/01. nmd.0000178038.87332.ec.

Morgan, H. L., Turner, D. C., Corlett, P. R., Absalom, A. R., Adapa, R., Arana, F. S., Pigott, J., Gardner, J., Everitt, J., Haggard, P. and Fletcher, P. C. (2011). Exploring the impact of ketamine on the experience of illusory body ownership, Biol. Psychiatry 69, 35-41. DOI:10. 1016/j.biopsych.2010.07.032.

O'Sullivan, N., de Bezenac, C., Piovesan, A., Cutler, H., Corcoran, R., Fenyvesi, D. and Bertamini, M. (2018). I am there ... but not quite: an unfaithful mirror that reduces feelings of ownership and agency, Perception 47, 197-215. DOI:10.1177/0301006617743392.

Paladino, M. P., Mazzurega, M., Pavani, F. and Schubert, T. W. (2010). Synchronous multisensory stimulation blurs self-other boundaries, Psychol. Sci. 21, 1202-1207. DOI:10.1177/ 0956797610379234.

Park, H.-D. and Blanke, O. (2019). Coupling inner and outer body for self-consciousness, $T r$. Cogn. Sci. 23, 377-388. DOI:10.1016/j.tics.2019.02.002.

Pelli, D. G. (1997). The VideoToolbox software for visual psychophysics: transforming numbers into movies, Spat. Vis. 10, 437-442. DOI:10.1163/156856897X00366.

Schabinger, N., Gillmeister, H., Berti, S., Michal, M., Beutel, M. E. and Adler, J. (2018). Detached and distracted: ERP correlates of altered attentional function in depersonalisation, Biol. Psychol. 134, 64-71. DOI:10.1016/j.biopsycho.2018.02.014.

Serino, A., Pizzoferrato, F. and Làdavas, E. (2008). Viewing a face (especially one's own face) being touched enhances tactile perception on the face, Psychol. Sci. 19, 434-438. DOI:10. 1111/j.1467-9280.2008.02105.x.

Serino, A., Giovagnoli, G. and Làdavas, E. (2009). I feel what you feel if you are similar to me, PLoS One 4, e4930. DOI:10.1371/journal.pone.0004930.

Seth, A. K. and Tsakiris, M. (2018). Being a beast machine: the somatic basis of selfhood, Tr. Cogn. Sci. 22, 969-981. DOI:10.1016/j.tics.2018.08.008.

Seth, A. K., Suzuki, K. and Critchley, H. D. (2011). An interoceptive predictive coding model of conscious presence, Front. Psychol. 2, 395. DOI:10.3389/fpsyg.2011.00395.

Sierra, M. (2009). Depersonalization: a New Look at a Neglected Syndrome. Cambridge University Press, Cambridge, UK.

Sierra, M. and Berrios, G. E. (2000). The Cambridge despersonalization scale: a new instrument for the measurement of despersonalization, Psychiatry Res. 93, 153-164. DOI:10. 1016/S0165-1781(00)00100-1.

Sierra, M. and David, A. S. (2011). Depersonalization: a selective impairment of self-awareness, Consc. Cogn. 20, 99-108. DOI:10.1016/j.concog.2010.10.018.

Sierra, M., Baker, D., Medford, N. and David, A. S. (2005). Unpacking the depersonalization syndrome: an exploratory factor analysis on the Cambridge depersonalization scale, Psychol. Med. 35, 1523-1532. DOI:10.1017/S0033291705005325.

Simeon, D. (2004). Depersonalisation disorder: a contemporary overview, CNS Drugs 18, 343 354. DOI:10.2165/00023210-200418060-00002.

Simeon, D. and Abugel, J. (2006). Feeling Unreal: Depersonalization Disorder and the Loss of the Self. Oxford University Press, Oxford, UK. 
Simeon, D., Guralnik, O., Hazlett, E. A., Spiegel-Cohen, J., Hollander, E. and Buchsbaum, M. S. (2000). Feeling unreal: a PET study of depersonalization disorder, Am. J. Psychiatry 157, 1782-1788. DOI:10.1176/appi.ajp.157.11.1782.

Tajadura-Jiménez, A. and Tsakiris, M. (2014). Balancing the "inner" and the "outer" self: interoceptive sensitivity modulates self-other boundaries, J. Exp. Psychol. Gen. 143, 736-744. DOI:10.1037/a0033171.

The GIMP team (2017). GNU Image Manipulation Program. Retrieved from https://www.gimp. org/.

Tottenham, N., Tanaka, J. W., Leon, A. C., McCarry, T., Nurse, M., Hare, T. A., Marcus, D. J., Westerlund, A., Casey, B. J. and Nelson, C. (2009). The NimStim set of facial expressions: judgments from untrained research participants, Psychiatry Res. 168, 242-249. DOI:10. 1016/j.psychres.2008.05.006.

Tsakiris, M. (2008). Looking for myself: current multisensory input alters self-face recognition, PLoS One 3, e4040. DOI:10.1371/journal.pone.0004040.

Tsakiris, M. (2010). My body in the brain: a neurocognitive model of body-ownership, Neuropsychologia 48, 703-712. DOI:10.1016/j.neuropsychologia.2009.09.034.

Zahavi, D. (2005). Subjectivity and Selfhood: Investigating the First-Person Perspective. MIT Press, Cambridge, MA, USA. 